\title{
A expansão do tratamento supervisionado (TS) para o controle da tuberculose na Paraíba (1999/2005)
}

\section{The expansion of the treatment observed (TS) for the control of tuberculosis in Paraiba (2000/2005)}

\section{La expansión del tratamiento supervisionado (TS) para el control de la tuberculosis en Paraíba (2000/2005)}

\author{
Gianne de Oliveira Rodrigues', Lenilde Duarte de Sá", J ordana de Almeida Nogueira'"', Lecidâmia \\ Cristina Leite Damascena ${ }^{\mathrm{V}}$, Anna Luiza Castro Gomes ${ }^{\mathrm{v}}$, Tereza Cristina Scatena Villa ${ }^{\mathrm{VI}}$
}

\section{RESUMO}

O tratamento supervisionado (TS) é um dos pilares da Estratégia DOTS (Directly Observed Treatment Short-course) e sua adoção vem sendo recomendada, para o controle da tuberculose (TB). Neste estudo objetivou-se analisar as potencialidades e fragilidades no processo de expansão do Tratamento Supervisionado (TS) nos seis municípios considerados prioritários para o controle da TB na Paraíba-PB/Brasil. Pesquisa de natureza qualitativa, que utilizou a entrevista semiestruturada com 21 profissionais envolvidos na implantação e expansão do DOTS nos seis municípios. Os dados foram analisados pela técnica de análise de conteúdo, modalidade temática. Como potencialidades foram identificadas: apoio dos gestores no processo de descentralização do TS para as Unidades de Saúde da Família (USF); compromisso dos profissionais e boa comunicação entre USF e referências. Dentre as fragilidades relatadas observa-se a falta de apoio dos gestores para garantir infra-estrutura e estímulo financeiro para o profissional; falta de qualificação profissional; alta rotatividade dos profissionais e centralização da distribuição de medicamento. Apesar dos esforços para implantação do TS, reconhece-se que sua ampliação esta condicionada a capacidade gerencial e ao compromisso político dos gestores.

Palavras chave: Tuberculose; Epidemiologia; Promoção da Saúde; Enfermagem em saúde Comunitária.

\section{ABSTRACT}

The supervised treatment (TS) is a pillar of the DOTS strategy (Directly Observed Treatment Short-course) and its adoption has been recommended for the control of tuberculosis (TB). The study aimed to analyze the strengths and weaknesses in the expansion process of the supervised treatment in six cities considered as priorities for the TB control in Paraíba-PB/Brazil. The qualitative research used semi-structured interview with 21 professionals involved in the implementation and expansion of DOTS in the cities, data were analyzed through thematic content analysis. As strengths it was identified: support to managers in the decentralization process of TS to the Family Health Units (USF); professionals' engagement and good communication between USFs and references. Among the reported weaknesses it is observed the lack of support from managers to ensure infra-structure and financial incentive to the professional; lack of professional qualification; high professional turnover and centralization of drug distribution. Despite the efforts for the TS implementation, it is recognized that its expansion is subject to the managerial ability and the managers' political commitment.

Key words: Tuberculosis; Epidemiology; Health Promotion; Community Health Nursing.

Enfermeira graduada pela Universidade Federal da Paraíba em 2007. Coordenadora do Programa Saúde da Família do Município de Condado - PE. Email: gianne.enf@hotmail.com.

" Profa. Dra. Dep.de Enfermagem de Saúde Pública e Psiquiatria da Universidade Federal da Paraíba e vinculada ao Programa de Pós-Graduação em EnfermagemPPGENF/UFPB. João Pessoa - PB. E-mail: lenildesa@yahoo.com.br.

III Profa. Dra. Dep.de Enfermagem Médico-Cirúrgica e Administração da Universidade Federal da Paraíba e vinculada ao Programa de Pós-Graduação em EnfermagemPPGENF/UFPB. João Pessoa - PB. E-mail: jal_nogueira@yahoo.com.br.

Iv Enfermeira graduada pela Universidade Federal da Paraíba em 2007. Enfermeira do Hospital Português - Recife/PE. Email: lecidamia@hotmail.com.

$\checkmark$ Mestre em enfermagem pelo Programa de Pós-Graduação em Enfermagem-PPGENF/UFPB. Enfermeira do Programa de Saúde da Família de João Pessoa - PB. E-mail: annaenf@gmail.com.

${ }^{\mathrm{V}}$ Enfermeira. Doutora em Enfermagem e Saúde Pública pela Escola de Enfermagem de Ribeirão Preto (EERP) da Universidade de São Paulo (USP). Ribeirão Preto/SP - Brasil. E-mail: tite@eerp.usp.br. 


\section{RESUMEN}

El tratamiento supervisado (TS) es uno de los pilares de la Estrategia DOTS (Directly Observed Treatment Short-course) y su adopción está siendo recomendada para el control de la tuberculosis (TB). En este estudio se objetivó analizar las potencialidades y fragilidades en el proceso de expansión del Tratamiento Supervisado (TS) en seis municipios considerados prioritarios para el control de TB en Paraíba-PB/Brasil. Es una investigación cualitativa que utilizó entrevista semiestructurada con 21 profesionales implicados en la implantación y expansión del DOTS en los seis municipios, se hizo el análisis de contenido temático de los datos. Como potencialidades fueron identificadas: apoyo de los gestores en el proceso de descentralización del TS para las

\section{NTRODUÇÃO}

Em 1993, a Organização Mundial de Saúde (OMS) declarou a situação da Tuberculose (TB) como emergência global propondo o Tratamento Diretamente Observável de Curta Duração (DOTS) como estratégia para seu controle. Esta recomendação visa reduzir a resistência medicamentosa, melhorar a adesão terapêutica, incrementar os percentuais de cura e, conseqüentemente, reduzir a incidência e a mortalidade por $\mathrm{TB}^{(1)}$.

Para que a implementação da estratégia DOTS seja efetiva, cinco pilares ou elementos essenciais devem ser observados: Detecção de casos pela microscopia; Regime de tratamento padronizado de, no mínimo, seis a oito meses para todos os casos confirmados a partir dos testes positivos de secreção, com tratamento direto observado ("DOT") pelo menos nos dois meses iniciais; Provisão regular de todos os medicamentos antituberculostáticos; Sistema padronizado e eficiente de registro e notificação de dados; e Compromisso político dos governos no suporte financeiro das atividades de controle da doença ${ }^{(2)}$.

Tratamento supervisionado

(DOTS) define-se, como a observação direta da tomada de medicamento para tuberculose pelo menos uma vez por semana durante o primeiro mês de tratamento, aliada à vontade política, à aquisição e distribuição regular de medicamentos e ao regular sistema de informação ${ }^{(3)}$. Implica, em uma reorganização
Unidades de Salud de la Familia (USF); compromiso de los profesionales y buena comunicación entre USFs y referencias. Entre las fragilidades relatadas se observa la falta de apoyo de los gestores para garantizar infraestructura y estímulo financiero para el profesional; falta de cualificación profesional; alta rotación de los profesionales y centralización de la distribución de medicamento. A pesar de los esfuerzos para implementación del TS, se reconoce que su ampliación está condicionada a la capacidad gerencial y al compromiso político de los gestores.

Palabras clave: Tuberculosis; Epidemiología; Promoción de la Saúde; Enfermería en salud comunitaria.

do trabalho que se coloca na prática como uma ação de enfoque terapêutico, que privilegia ações educativas no âmbito familiar e a aproximação à realidade social dos pacientes ${ }^{(4)}$.

Nos últimos dez anos, a estratégia DOTS, vem sendo implementada pelos 184 países que acumulam $99 \%$ dos casos estimados de TB. Em 2006, 4,9 milhões de casos novos foram notificados por programas que aderiram ao DOTS, representando $98 \%$ do total de casos registrados mundialmente ${ }^{(2)}$.

No Brasil, em 1998, ano de implantação da estratégia DOTS, apenas 3\% da população era coberta pelo DOTS, sendo que somente $27,6 \%$ dos estabelecimentos do sistema público de saúde integravam o programa, em sua maioria, centros de saúde especializados ${ }^{(3)}$. Nos dois anos subseqüentes $(1999,2000)$, sua implementação foi tímida alcançando cobertura populacional de $7 \%^{(2)}$.

Em decorrência às mudanças estabelecidas pelo Plano Nacional de Controle da Tuberculose (PNCT) - 2001-2005, consoante às diretrizes do Sistema Único de Saúde (SUS) que propõe a descentralização das ações de saúde para o âmbito da atenção básica, observa-se expansão gradual da inserção das ações de controle da TB pelas Equipes de Saúde da Família (ESF). Em 2006, a cobertura populacional pela estratégia DOTS alcançou $86 \%{ }^{(2)}$.

Embora a expansão das ações de controle da TB, por meio da estratégia DOTS, seja um 
evento que vem sendo incorporado pela atenção básica, verifica-se que, em alguns municípios, o acompanhamento dos casos de TB, ainda permanece centralizado nas Unidades de Referência (UR) e que apenas algumas ações, tais como, o diagnóstico e o Tratamento Supervisionado (TS) vêm sendo descentralizados para as ESF ${ }^{(5)}$. Esse problema alia-se a outros entraves inerentes ao atual sistema público de saúde, como a escassez de recursos humanos e financeiros ${ }^{(6)}$. O Ministério da Saúde admite a necessidade de investimentos na qualificação dos serviços de saúde, na capacitação de recursos humanos para desenvolver as atividades de vigilância, avaliação e controle nos municípios brasileiros e em especial nos municípios prioritários para o $\mathrm{PNCT}^{(7)}$.

Reconhece-se que a efetivação da estratégia DOTS requer compromisso político do gestor local para ampliar capacidade e o desempenho das ESF. Entende-se nesta direção por capacidade, os recursos básicos necessários para o desenvolvimento das ações (organização da rede para apoio diagnóstico, sistema de informação, distribuição regular dos medicamentos, insumos) e por desempenho, a reorganização do processo de trabalho como uma ação de enfoque terapêutico, que privilegie as ações educativas no âmbito familiar e a aproximação à realidade social dos pacientes ${ }^{(4)}$.

No Estado da Paraíba, a implantação da estratégia DOTS foi iniciada em 1999, estando, desde então, articulada com a política de municipalização e, principalmente com as ações voltadas à reorganização dos serviços de atenção básica ${ }^{(8)}$. Nos seis municípios paraibanos considerados prioritários para o controle da TB pelo PNCT, a responsabilidade das ações relacionadas ao DOTS tem sido transferida para as ESF, principalmente, no que concerne à identificação dos usuários, à notificação de casos e ao $\mathrm{TS}^{(5)}$.

Não obstante, observam-se alguns fatores positivos e/ou negativos, intimamente relacionados ao processo de trabalho das ESF e dos coordenadores locais de Programa de Controle da Tuberculose $(\mathrm{PCT})^{(5)}$. Identificar esses fatores significa considerar $\mathrm{O}$ monitoramento das ações sob o olhar da vigilância em saúde e, neste caso, permanentemente reconhecer problemas para poder esclarecê-los, minimizá-los ou resolvêlos.

O referencial teórico deste estudo ancorase na definição da coordenação como uma dimensão da Atenção Primária em Saúde (APS). Por APS compreende-se "[...] aquele nível de um sistema de serviço de saúde que oferece a entrada no sistema para todas as novas necessidades e problemas, fornece atenção sobre a pessoa (não direcionada para a enfermidade) no decorrer do tempo, fornece atenção para todas as condições, exceto as muito incomuns ou raras, e coordena ou integra a atenção fornecida em algum outro lugar ou por terceiros" ${ }^{\prime(9)}$. Em atenção ao conceito citado, uma vez que, na política de saúde brasileira se preconiza que o controle da TB seja feito na esfera da APS, tendo a estratégia Saúde da Família como o lócus inicial de entrada do usuário no sistema de saúde, se faz necessário que essa estratégia funcione como eixo articulador do suprimento das necessidades dos doentes com TB na rede de serviços de saúde.

$\mathrm{Na}$ perspectiva do processo do cuidado em rede, principalmente quando se trata do usuário com TB, vale considerar as conseqüências negativas que podem derivar da falta de continuidade do tratamento. Nesse enfoque, reconhece-se que o êxito do TS depende do modo como se gerencia o sistema de saúde e a própria sustentabilidade do DOTS. Nessa perspectiva, todos os seus pilares devem ser observados, dentre eles a coordenação da assistência, como dimensão da APS, descrita como instrumento gerencial de integração de todos os serviços relacionados à saúde, sendo a organização do trabalho realizada com diversos profissionais que possuem responsabilidades distintas, porém compromissos iguais na produção dos serviços de saúde ${ }^{(9)}$.

Reconhecendo a importância da coordenação como uma das dimensões da APS diretamente relacionada com a continuidade do tratamento da TB e considerando as explanações realizadas, o objetivo deste estudo foi analisar, sob a ótica dos profissionais envolvidos com o PCT, as potencialidades e fragilidades encontradas no processo de 
expansão do TS nos seis municípios prioritários para o controle da Tuberculose na Paraíba.

\section{METODOLOGI A}

O presente estudo constitui parte da pesquisa intitulada "Situação da implantação do DOTS em algumas regiões do Brasil: Histórico e peculiaridades de acordo com as características regionais", realizada pela Área de Estudos Operacionais da Rede Brasileira de Pesquisa em Tuberculose - REDE-TB, cujo objetivo foi descrever o histórico e a situação da implantação da estratégia DOTS no controle da tuberculose na macrorregião Centro Oeste, nos Estados de São Paulo e Paraíba; cidades de Recife, Rio de Janeiro, Ribeirão Preto, São José do Rio Preto e Porto Alegre.

Este estudo tem como recorte espacial geral o Estado da Paraíba, especificamente, os seis municípios considerados prioritários pelo Ministério da Saúde para o controle da tuberculose neste Estado, sendo eles: J oão Pessoa, Campina Grande, Bayeux, Santa Rita, Patos e Cajazeiras. O primeiro refere-se a capital. Em seguida é citado o segundo maior município paraibano em número de habitantes e geração de renda; o terceiro e o quarto integram a região metropolitana da Grande J oão Pessoa e os dois últimos estão localizados no Alto Sertão da Paraíba.

O período 1999 a 2005, foi considerado relevante para reunir informações sobre o TS, já que em 1999 a estratégia DOTS começou a ser implantada na Paraíba.

Optou-se, neste estudo pela abordagem qualitativa. Utilizou-se como recurso para coleta de dados a técnica da entrevista semiestruturada que combina perguntas abertas e fechadas. Os dados foram coletados entre os meses de outubro e novembro de 2005 e contou com a participação de 21 profissionais, sendo o critério de escolha dos sujeitos o fato dos profissionais terem participado do processo de implantação da estratégia DOTS nos municípios prioritários. A seleção dos atores inclui gestores de saúde, como coordenadores do PCT, membros da equipe de saúde que realizam o TS, coordenadores de vigilância epidemiológica e diretores de centros de referências e profissionais de saúde que realizam o TS. Os entrevistados foram codificados com a letra $P$ (profissionais), seguido de números arábicos, em ordem progressiva de P1 a P21.

Para viabilizar a coleta de dados, inicialmente, os pesquisadores responsáveis pelo estudo na Paraíba fizeram contatos com os coordenadores municipais do PCT e do estado, a fim de identificar os atores que participaram do processo de implantação da estratégia DOTS, facilitando assim a aproximação dos pesquisadores com os profissionais. As entrevistas foram previamente agendadas e realizadas de acordo com a disponibilidade de cada profissional participante, em residências e/ou em seus locais de trabalho.

Os dados foram analisados a partir da técnica de análise de conteúdo, modalidade temática, a qual consiste em descobrir os núcleos de sentido que compõem uma comunicação cuja presença ou freqüência signifiquem alguma coisa para o objetivo analítico visado ${ }^{(10)}$. A técnica da análise temática se desdobra em três etapas: préanálise, exploração do material e tratamento, inferência e interpretação dos dados.

A etapa da pré-análise corresponde à organização das idéias propriamente ditas, de forma sistemática, de maneira a conduzir um esquema preciso do desenvolvimento das operações sucessivas, em um plano de análise. Esse plano foi iniciado pela organização do material disponível para a análise, no caso as entrevistas transcritas na íntegra, seguido do processo de leitura flutuante.

Com relação à exploração do material, nesta etapa, em conformidade com a recorrência das informações coletadas, foram destacadas as unidades de registro e a temática em relevo. Em seguida, procurou-se a articulação entre as falas, conteúdos convergentes, divergentes e repetidos, recortando-se os extratos das falas em cada um dos núcleos de sentido identificados. Isso permitiu eleger as unidades temáticas contidas no conjunto dos discursos, em um movimento de classificação. Foi eleito como Unidade Temática Central o processo de expansão do Tratamento Supervisionado para os serviços de 
saúde, cujo desmembramento permitiu a identificação dos núcleos de sentidos determinantes para a construção de duas categorias empíricas. A primeira trata das potencialidades observadas no processo de expansão do TS nos municípios prioritário da Paraíba. A segunda enfoca as fragilidades do referido processo.

Por fim, na etapa de tratamento, inferência e interpretação dos dados, realizou-se a análise dos dados propriamente ditos. Ou seja, a partir dos resultados obtidos nas etapas anteriores, foram empregadas inferências e interpretações à luz da fundamentação teórica e dos pressupostos que nortearam esta investigação.

Considerando os aspectos éticos, ressaltase que a pesquisa foi realizada segundo a Resolução $196 / 96^{(11)}$. O projeto para a realização do estudo foi submetido ao Comitê de Ética em Pesquisa do Centro de Ciências da Saúde, sendo a sua execução aprovada pelo Protocolo 823/06.

\section{RESULTADOS E DISCUSSÃO}

A Unidade Central identificada será aqui discutida por meio da categoria: "As potencialidades e fragilidades no processo de expansão do TS", a qual expressa a percepção dos profissionais envolvidos com a implantação e expansão do TS nos municípios prioritários do Estado da Paraíba.

Dados do relatório da Organização Mundial da Saúde (OMS) mostram que, dentre os atuais entraves para a expansão do DOTS, cinco são merecedores de destaque: equipes com curto treinamento; lacuna no compromisso político; serviços laboratoriais deficientes; gerenciamento inadequado dos casos de multirresistência (MDR) e a co-infecção $\mathrm{TB} / \mathrm{HIV}^{(12)}$. No Brasil, os poucos trabalhos publicados sobre o DOTS voltam-se para aspectos que analisam a eficácia/eficiência da estratégia, com pouca ênfase nos aspectos operacionais propriamente ditos, ou seja, na descrição de elementos que enfoquem fragilidades e potencialidades ${ }^{(13)}$.

Sabe-se que para se alcançar o controle da TB, são necessárias ações que ultrapassem os artifícios diretamente voltados à administração da quimioterapia, sendo indispensável mudanças qualitativas de caráter político, administrativo e organizacional, envolvendo os serviços de saúde ${ }^{(14)}$. Nesse sentido, a implementação do DOTS no estado da Paraíba, apesar de contribuir para mudanças positivas de indicadores, o que chama atenção para as potencialidades desta estratégia, por outro lado, a sua situação apresenta desafios, aqui expressos como fragilidades.

Alguns problemas do DOTS estão vinculados à política que conforma o sistema público de saúde dos municípios, como a escassez de recursos humanos e financeiros, os quais dificultam o emprego desta estratégia para a maioria dos doentes que fazem tratamento de tuberculose ${ }^{(6)}$. Muitos destes problemas provêm de uma débil vontade política para melhorar o sistema de saúde e a resposta social necessária para o controle da doença $^{(14)}$.

A atuação técnica dos gestores no SUS é permeada por variáveis da política de saúde e pelas necessidades da população. Nesse sentido, reconhecendo na dimensão de coordenação da APS relacionada ao exercício da gestão, se buscou analisar as debilidades e as fortalezas da expansão do TS, articulado aos demais pilares da estratégia DOTS, nos municípios prioritários para o controle da TB na Paraíba.

O apoio e o compromisso dos gestores, na forma da ordenação e planejamento das ações políticas voltadas ao controle da TB são registrados nos discursos de alguns profissionais como um aspecto facilitador no processo de sustentabilidade e ampliação da estratégia DOTS. Aponta-se, por exemplo, a garantia de pontos cruciais para o êxito da estratégia, como a capacitação dos profissionais que agora assumem a responsabilidade direta do cuidado ao doente de TB.

É justamente a gente tendo disposição para o trabalho, ter o compromisso profissional de todos, e ter o apoio do gestor. (P6)

Eu acho que é o compromisso. Como eu te disse do gestor. O gestor é comprometido, é interessado, ele quer que as pessoas sejam capacitadas, porque ele não entende que você 
possa implantar ou acompanhar determinado programa sem a capacitação. (P13)

Entretanto, o compromisso político dos gestores paraibanos, mostra-se insuficiente quando se analisa o fator financiamento de ações. As falas dos profissionais envolvidos com - DOTS em dois municípios, sendo um pertencente à região metropolitana da Grande João Pessoa e outro a mesorregião da Borborema, revelam o escasso apoio financeiro necessário para garantir infra-estrutura e estímulo para o profissional, o que concorre para dificultar a expansão da estratégia:

É justamente com relação, muitas vezes, ao recurso financeiro. Isso dificulta muito, o nosso desempenho. Até para a gente pode, aumentar, implementar e implantar em novas Unidades. (P6)

(...) Porque o financeiro pesa, porque a gente gostaria de ter em cada equipe, em todas as equipes, aquela coisa básica do café com leite e bolachas na hora do atendimento supervisionado. [...] a gente queria ter uma coisa mais atrativa para os pacientes (...). ( P8) Precisa conscientizar o profissional de saúde e para conscientizar o profissional de saúde vai ter que dar a estabilidade de emprego e além do mais a compensação financeira. (P11)

A insuficiência de recursos financeiros, bem como a ineficiência na utilização desses, é entre outros aspectos, enfatizada pela Organização Mundial de Saúde (OMS) como importante obstáculo à expansão do DOTS ${ }^{(12)}$. Portanto, cabe nesse momento acrescentar, que o efetivo compromisso político para o controle da TB consiste na construção da consciência do problema da doença e da compreensão dos benefícios de seu real controle, o que abrange, além da individualidade do paciente, a provisão de soluções custo-efetivas ${ }^{(15)}$.

Em um dos municípios do Alto Sertão, por sua vez, pôde-se ressalvar a omissão da gestão quanto ao controle e avaliação das ações de controle da doença, uma vez que, neste município, não se observa a provisão de recursos materiais, para garantir a qualidade das ações que envolvem o controle da doença:
É porque muitas vezes devido, a ser uma cidade grande e a secretaria não dispõe de uma frota de carros a altura para que possa assim... muitas vezes a gente quer fazer uma supervisão numa Unidade e a gente não tem o carro e isso dificulta um pouco. (P14)

Deve-se aqui ressaltar que a perspectiva da supervisão e a avaliação das ações de saúde podem contribuir para as políticas públicas como ferramenta do planejamento e da gestão, podendo trazer inovações no processo de implementação da política de descentralização do setor saúde(16). O PNCT para 2001-2005 considera a avaliação local permanente como única forma capaz de corrigir falhas, em tempo de evitar os danos que tratamentos mal conduzidos geram para a população e para as autoridades sanitárias do País.

Sobre a prestação direta de serviços de saúde vale salientar a alusão feita à comunicação entre as unidades onde se dá o TS e as UR de cada município, principalmente no que diz respeito à retaguarda laboratorial e a provisão de medicamentos. $\mathrm{Na}$ seqüência, segue os discursos relacionados a essa questão nos municípios de Borborema e do Alto Sertão, respectivamente:

Especialmente a facilidade de comunicação, é, como eu falei anteriormente, se a gente precisa de um exame como o RX que demora um pouco mais, a gente liga para a secretaria de saúde e ela procura agilizar através dos gestores de programa de TB (...). (P10)

A facilidade que eu tenho na verdade eu recebo de lá do posto de referência toda a medicação [...] E também qualquer problema, sempre que tenho alguma dificuldade, entro em contato com a referência. (P17)

Todavia, a ocorrência dos eventos supracitados, essenciais para assegurar a acessibilidade e a qualidade no diagnóstico e tratamento da TB, por vezes, esbarra em limitações operacionais, como é o caso de outro município do Alto Sertão onde há a disponibilização dos tuberculostáticos, entretanto, a centralização no fornecimento 
Rodrigues GO, Sá LD, Nogueira JA, Damascena LCL, Gomes ALC, Villa TCS. A expansão do tratamento supervisionado (TS) para o controle da tuberculose na Paraíba (1999/2005). Rev. Eletr. Enf. [Internet]. 2008; 10(3):632-42. Available from: http://www. fen.ufg. br/revista/v10/n3/v10n3a09.htm.

desses medicamentos, é apontada pelos entrevistados como dificuldade à operacionalização do TS.

As dificuldades nesse processo é a questão da redistribuição da medicação. Nós temos que ir buscar essa medicação, ela não vem para seis meses, então mensalmente nós estamos indo lá para pegar essa medicação. (P21)

A portaria $n^{\circ} 2.646 / \mathrm{GM}$, de 2004, garante o repasse de bônus anual mediante a apresentação de um plano de trabalho elaborado pelos municípios. O MS distribuiu o recurso para os municípios prioritários em 2004 mediante um Plano de Aplicação que divide a distribuição do recurso em cinco áreas: capacitação, educação em saúde, vigilância epidemiológica, laboratório e supervisão(17). Esse repasse de recursos federais aos municípios prioritários na forma de bonificação foi mencionado como grande incentivo ao interesse de gestores no município da mesorregião da Borborema.

Uma facilidade vai ser a portaria ministerial, que há este repasse fundo a fundo, e que realmente os gestores municipais na captura de recursos para seu município ele se sente mais interessado porque o recurso é maior do que o auto-administrado [...] Tem uma grande facilidade para implantação, há o interesse do gestor municipal e também da equipe do PSF (..). ( (P12)

Outra responsabilidade inerente à gestão, no que diz respeito à expansão do DOTS, é a mobilização dos serviços de saúde, usuários e a sociedade em geral para incorporação do real sentido da estratégia. De acordo com a OMS a participação comunitária na atenção à TB e a necessidade de enfatizar a promoção no paciente são necessárias para melhorar tanto o acesso como a utilização dos serviços de saúde ${ }^{(12)}$. Dessa forma, pode-se afirmar que a preocupação com o controle da doença não pode ser voltada apenas a ingesta do medicamento, mas também para a educação e a participação, ações essas fundamentais para o processo de adesão do paciente.
Neste sentido o acolhimento e o vínculo constituem importantes princípios no acompanhamento dos casos de tuberculose, haja vista que estabelece uma relação de confiança entre o profissional de saúde e paciente, que passa a compreender a significância de seu tratamento(16)

O paciente é tratado perto da sua casa, o paciente toma o medicamento diariamente, ele tem um lanche após o medicamento, ele é feito alguma consulta, dependendo de algum problema, e também é feito o processo de habitação e saúde. (P20)

Um dos principais problemas encontrado pelo PCT refere-se à adesão dos pacientes com tuberculose à terapêutica oferecida. A nãoadesão ao tratamento é apontada como uma das graves falhas no programa para combater a doença ${ }^{(18)}$. Assim como a não adesão, o abandono do tratamento concorre para elevar a incidência de bacilos multi-droga resistente (MDR) que, associada à desigualdade social, ao advento da AIDS, o envelhecimento da população e os grandes movimentos migratórios, acentuam mundialmente a gravidade da situação atual da TB.

Estudos revelam que os primeiros dois a três meses de tratamento de TB são os períodos nos quais ocorrem a maioria dos abandonos, evidenciando a importância de adotar medidas eficazes desde o princípio do tratamento para reduzir este problema ${ }^{(6)}$. Os motivos relacionados a este evento são complexos e diversificados, estando relacionados ao paciente, ao tratamento e à organização dos serviços de saúde ${ }^{(19)}$.

Estudos relacionados à não adesão ao tratamento por parte dos doentes demonstram a existência de inúmeras variáveis demográficas e sócio-econômicas interferindo nesse processo. Entretanto, na prática, o comportamento sanitário da maioria das pessoas é imprevisível e não confirmam as expectativas dos serviços de saúde de que sigam as recomendações médicas $^{(18)}$.

Uma associação já conhecida e amplamente divulgada na literatura diz respeito ao aumento da probabilidade de abandono do 
tratamento por doentes considerados dependentes de álcool. Em estudo realizado em $2005^{(6)}$, verificou-se que doentes de TB alcoolistas possuíam 3,3 vezes mais chance de abandono de tratamento quando comparados aos não alcoolistas. Na realidade dos municípios aqui estudados, o fato do usuário ser etilista é reconhecido como um problema relacionado à continuidade do tratamento.

(...) a única dificuldade que tem é se o paciente é etilista, estes são difíceis, porque eles sentem mal-estar por conta da medicação, aí pensam que podem continuar bebendo e se medicando e isto eles dificultam mesmo, a gente tem que estar em cima. (P3)

A gente encontra resistência como eu lhe falei. Por exemplo, tem paciente que teve nenhuma dificuldade, ele aderiu completamente ao tratamento e tudo, mas nós temos diversos pacientes que têm dificuldades, são os alcoólatras, que a gente tem dificuldade de continuar o tratamento, porque às vezes eles não aceitam, abandonam, precisa a gente ir lá buscar, trazer novamente. (P17)

A sensibilização e o envolvimento da equipe de saúde, promovendo acolhimento e vínculo, são os elementos chaves no processo de adesão do usuário ao tratamento da tuberculose ${ }^{(16)}$. O vínculo permite a formação de laços de empatia e solidariedade entre os profissionais de saúde e o paciente, ocorrendo, por vezes, um processo de transferência, no qual um passa a representar algo importante na vida do outro. E assim, cria-se um espaço aberto ao diálogo e o paciente passa a entender a significância de estar inserido no TS. Dessa forma, pode-se inferir que a TB é uma doença presente e dependente de uma mudança de concepções, seja em relação à reformulação de políticas que enfatizam a tuberculose como prioridade, ou

ao gerenciamento/coordenadorias ou, ainda, ao envolvimento das equipes responsáveis pela assistência aos portadores de $\mathrm{TB}^{(13)}$.

Considerando o envolvimento dos profissionais, e seus efeitos no processo de ampliação da estratégia DOTS no estado da Paraíba, verifica-se que, de modo geral, há uma tendência à falta de compromisso por parte das equipes, o que pode ser identificado como o maior obstáculo à expansão da estratégia na fala da coordenadora do DOTS na referencia estadual:

(...) a falta de compromisso até de alguns profissionais em querer assumir, eu acho que essa é a grande dificuldade.(P1)

Seguindo a mesma linha de raciocínio e confirmando a inferência da coordenação estadual, os profissionais de quatro dos seis municípios prioritários apontam problemas inerentes aos profissionais como responsáveis pela limitação a ampliação do DOTS, por motivos relacionados à falta de compromisso/sensibilização, ausência de capacitação/ treinamento, a rotatividade e a critérios de seleção dos recursos humanos e, ainda o preconceito e o medo que mostram em relação ao doente.

Sobre o processo de envolvimento da equipe de saúde no cuidado ao portador de TB, observamos a dificuldade dos profissionais em incorporar intervenções no processo de trabalho que contemplem a singularidade, necessidades e problemas do paciente e família sob tratamento para $\mathrm{TB}^{(17)}$. Desse modo, a sensibilização bem como o envolvimento da equipe, com o conseqüente estabelecimento do vínculo profissional/usuário podem ser considerados como elementos imprescindíveis na operacionalização do TS e controle da TB. Os profissionais do município da Borborema foram os que mais reconheceram este aspecto do processo terapêutico:

(...) e a falta de sensibilização, como eu já falei a gente tem que conseguir sensibilizar a rede de recursos humanos. (P8)

É a vontade, a vontade de você fazer as coisas acontecerem. Muitas vezes as pessoas estão sensibilizadas, capacitadas, são orientadas, no caso para usar a Estratégia DOTS, mas usam um monte de desculpas, um monte de dificuldade, então nesse caso é a questão da vontade de fazer. (P9)

A dificuldade é a falta de compromisso por parte de alguns profissionais de saúde $[\ldots]$, e de 
sensibilização sobre o que é realmente DOTS. (P12)

A proposta de levar saúde para todos, reformulando o fantasma social da doença, traz consigo a necessidade de definir estratégias de capacitação dos profissionais que estarão à frente desse processo, objetivando transformações no processo de trabalho, e a orientação destes para uma constante melhoria da qualidade das ações e serviços da saúde. Para tanto, esses profissionais devem ser qualificados de modo a desenvolver, satisfatoriamente, as ações de diagnóstico, tratamento, registro e notificação e outras atividades para cumprirem eficazmente suas responsabilidades específicas com apropriada supervisão ${ }^{(7)}$.

Embora se observe apoio por parte dos gestores para qualificar os trabalhadores da saúde envolvidos com o controle da TB, reconhece-se que essa ação precisa ser intensificada visando a qualidade de desempenho dos profissionais das ESF no cuidado ao portador de TB no Estado da Paraíba, como pontua um colaborador do município da região da Borborema.

As dificuldades como já comentamos seria de capacitar melhor os profissionais das unidades de saúde, porque foi descentralizado, estão sendo acompanhados, supervisionados não só o paciente como a equipe, a gente está acompanhando também os profissionais, daí eu acho que precisa uma capacitação melhor dos profissionais, para que realmente facilite 0 acompanhamento e o diagnóstico dos pacientes sintomáticos respiratórios. (P18)

No que diz respeito a qualificação dos trabalhadores da saúde para atuar no controle da TB na Paraíba, embora se reconheça a sua importância, em um dos municípios prioritários desse estudo, a secretaria de saúde do município, limita em 05 (cinco) dias por ano o direito do profissional de saúde ausentar-se para capacitação(20).

Embora os profissionais que participaram da implantação do DOTS ou que deram sua parcela de contribuição para a sustentabilidade desta estratégia nos seis municípios prioritários apontem algumas debilidades no processo de ampliação do TS, observam-se aspectos que revelam potencialidades da estratégia.

Em parte do período estudado, considerando sobretudo 1999 - ano da implantação da estratégia DOTS na Paraíba observa-se que os indicadores referentes à TB nos municípios prioritários sofreram alterações positivas. A taxa de cura que era de $63,7 \% \mathrm{em}$ 1999, alcançou 74\% em 2003. Verifica-se também a diminuição dos índices de abandono, que em 1999 era de $14,8 \%$ e em 2003 caiu para $5,8 \%{ }^{(5)}$. Os exemplos citados reafirmam a viabilidade da estratégia para o controle da TB e apontam para o envolvimento dos profissionais na implantação, sustentabilidade e expansão do TS, principalmente, por parte daqueles que atuam no âmbito da APS.

\section{CONSI DERAÇÕES FI NAIS}

A respeito das potencialidades identificadas no processo de expansão do DOTS na Paraíba pode-se fazer referência ao apoio dispensado pelos gestores em implantar a estratégia, garantindo a execução de pontos essenciais para seu êxito como a capacitação profissional e a provisão da medicação. O envolvimento por parte dos profissionais com o TS, a boa comunicação entre as USF que fazem o TS e as UR e o fato da expansão do DOTS estar acontecendo de forma articulada às USF, também são reconhecidos como potencialidades para a sustentabilidade da estratégia, sendo fortalecedoras do cuidado ao doente de TB na perspectiva da APS.

No que concerne às fragilidades no processo de ampliação do DOTS nos municípios paraibanos - e consequentemente a expansão do TS -, foram identificados problemas inerentes à gestão, aos doentes e aos profissionais, merecendo destaque: a falta de apoio financeiro por parte dos gestores para garantir a infra-estrutura necessária à realização do TS - como a alimentação e a viabilização do deslocamento dos doentes, e ainda o estímulo financeiro para o profissional-; a resistência à adesão ao tratamento por parte dos usuários; a falta de qualificação e sensibilização profissional para atuação no 
cuidado ao doente; a rotatividade $e$ as especialidades dos profissionais das USF e também a centralização no fornecimento dos medicamentos anti-tuberculose.

Considerando o conceito do atributo coordenação na APS, reconhece-se que as fragilidades encontradas influenciam de forma negativa a integração entre os serviços da rede de saúde, dos quais necessitem o doente de TB, bem como a organização do trabalho realizado por diversos profissionais envolvidos com 0 controle da doença.

As fragilidades apontadas no estudo demandam reflexões pelas coordenações estaduais e municipais do PCT em conjunto com os profissionais das ESF, representantes dos usuários e dos centros de formação e capacitação de trabalhadores envolvidos com o TS, na perspectiva de não apenas discutirem os problemas inerentes à sustentabilidade da estratégia DOTS, mas sobretudo participarem do planejamento das políticas e das ações para controle da TB na Paraíba.

\section{REFERÊNCI AS}

1. Organización Panamericana de la Salud. Reunión de Jefes de Programas Nacionales de Tuberculosis (PNT) de las Américas: informe final. Ciudad de México: Organización Panamericana de la Salud; 2004.

2. World Health Organization (WHO). Global tuberculosis control: surveillance, planning, financing. Geneva: World Health Organization; 2008.

3. Ministério da Saúde; Fundação Nacional de Saúde, Centro de referência Prof. Hélio Fraga. Sociedade Brasileira de Pneumologia e Tisiologia. Controle da Tuberculose - Uma proposta de Integração Ensino-Serviço. 5a edição. Rio de Janeiro (Brasil): FUNASA/CRPHF/SBPT; 2002.

4. Muniz JN, Palha PF, Monroe AA, Gonzáles RIC, Ruffino Netto A, Villa TCS. A Incorporação da busca ativa de sintomáticos respiratórios para o controle da tuberculose na prática do agente comunitário de saúde. Ciência e Saúde Coletiva. 2005; 10(2): 315-21.

5. Sá LD, Figueiredo TMRM, Lima DS, Andrade MN, Queiroga RP, Cardoso MAA, et al. A experiência da implantação da estratégia DOTS em seis municípios paraibanos. In: RuffinoNetto A, Villa TCS. Tuberculose: implantação do DOTS em algumas regiões do Brasil: histórico e peculiaridades regionais. Ribeirão Preto: FMRP/REDE TB-USP; 2006. p. 141-66.

6. Bergel FS, Gouveia N. Retornos freqüentes como nova estratégia para adesão ao tratamento de tuberculose. Revista de Saúde Pública. 2005; 39(6): 898-905.

7. Ministério da Saúde. Tuberculose: Guia de vigilância epidemiológica. Brasília: Ministério da Saúde; 2002.

8. Lima DS. Plano de trabalho, em parceria com os gestores municipais dos municípios prioritários de: João Pessoa, Bayeux, Santa Rita, Campina Grande, Patos e Cajazeiras a ser cumprido em 2005-2006. João Pessoa: Secretaria de Estado da Saúde; 2005.

9. Starfield B. Atenção primária: equilíbrio entre necessidades de saúde, serviços e tecnologia. Brasília: Ministério da Saúde; 2002.

10. Minayo MCS. O Desafio do Conhecimento: pesquisa qualitativa em saúde. São Paulo/Rio de J aneiro: Hucitec; 2007.

11. Ministério da Saúde; Comissão Nacional de Ética em Pesquisa, Conselho Nacional de Saúde. Resolução № 196/96 - Normas regulamentadoras de pesquisa envolvendo seres humanos. Brasília (Brasil): Ministério da Saúde; 1996.

12 World Health Organization (WHO). Global tuberculosis control: surveillance, planning, financing. Geneva: World Health Organization; 2005.

13. Ruffino-Netto A, Villa TCS. Tuberculose: Implantação dos DOTS em algumas regiões do Brasil: histórico e peculiaridades regionais. Ribeirão Preto: FMRP/REDE-TB; 2006.

14. Monroe AA, Cardozo RIG, Palha PF, Sassaki $\mathrm{CM}$, Ruffino Netto A, Vendramini SHF, et al. Envolvimento de equipes da atenção básica à saúde no controle da tuberculose. Revista da Escola de Enfermagem da USP. 2008; 42(2): 262-68.

15. Andrade $M N$. O envolvimento dos gestores de saúde na implantação e sustentabilidade da estratégia DOTS nos municípios prioritários para o controle da tuberculose na Paraíba [dissertação]. [João Pessoa]: Programa de PósGraduação em Enfermagem/UFPB; 2006. 
16. Arcêncio RA, Oliveira MF, Cardoso Gonzáles RI, Pinto IC, Ruffino Netto A, Villa TCS. City tuberculosis control coordinators' perspectives of patient adherence to DOT in São Paulo State, Brazil, 2005. The International Journal of Tuberculosis and Lung Disease. 2008; 12(5): 527-31.

17. Vendramini SHF, Villa TCS, Palha PF, Monroe AA. Tratamento supervisionado no controle da tuberculose em uma unidade de saúde de Ribeirão Preto: a percepção do doente. Boletim de Pneumologia Sanitária. 2002; 10(1): 5-12..

18. Costa JSD. Controle epidemiológico da tuberculose na cidade de Pelotas, Rio Grande do Sul, Brasil: adesão ao tratamento. Cadernos de Saúde Pública. 1998; 14(2): 409-15.

19. Sá LD, Souza KMJ, Nunes MG, Palha PF, Nogueira JA, Villa TCS. Tratamento da tuberculose em unidades de saúde da família: histórias de abandono. Texto e Contexto Enfermagem. 2007; 16(4): 712-8.

20. Silva ACO, Sousa MCM, Nogueira JA, Motta MCS. Tratamento supervisionado no controle da tuberculose: potencialidades e fragilidades na percepção do enfermeiro. Revista Eletrônica de Enfermagem [Internet]. 2007 [cited 2008 jul 17]; 9(2):402-16. Available from: http://www.fen.ufg.br/revista/v9/n2/pdf/v9n2a 09.pdf

Artigo recebido em 07.12.07

Aprovado para publicação em 30.09.08 